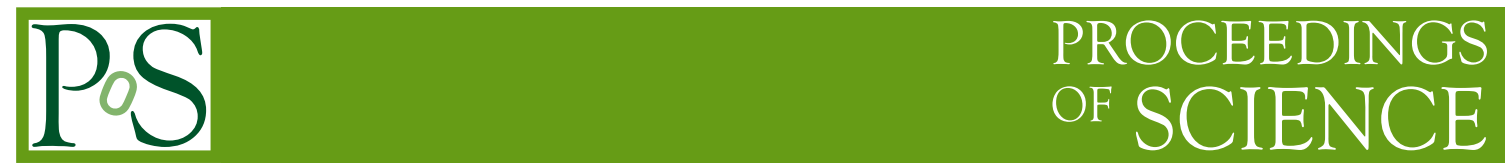

NNLO QCD corrections to $b \rightarrow u W^{*}$

\title{
Zhaoting Pan*
}

${ }^{\dagger}$ Laboratoire de Physique Subatomique et de Cosmologie, Université Joseph

Fourier/CNRS-IN2P3/INPG, F-38026 Grenoble, France

E-mail: zhaoting.pan@lpsc.in2p3.fr

We present the reduction to the Master Integrals (MIs) of the NNLO QCD corrections to the decay process $b \rightarrow u W^{*}$, where $\mathrm{b}$ and $\mathrm{u}$ are a massive and a massless quark, respectively, while $W^{*}$ is an off-shell charged weak boson. We focus on the Feynman diagrams for the real emission. We use the Cutkosky rules and the Laporta algorithm applied to the cut diagrams, as suggested for the Higgs production by Anastasiou and Melnikov.

The 2011 Europhysics Conference on High Energy Physics, EPS-HEP 2011,

July 21-27, 2011

Grenoble, Rhône-Alpes, France

\footnotetext{
* Speaker.

†This work was partly supported by the initiative "Projets de Physique Théorique de l'IN2P3".
} 


\section{Introduction}

The measurements of inclusive semileptonic B meson decays $\overline{\mathrm{B}} \rightarrow X_{u} l \bar{v}_{l}$ allow a precise determination of the CKM matrix element $\left|V_{u b}\right|$, which constitutes an important input parameter for many analyses in flavour physics.

The total decay rates of the B meson are described by a local Operator Product Expension in inverse powers of the b-quark mass $m_{b}$. To the leading order in $1 / m_{b}$, the total $\mathrm{B}$ meson decay rate is equivalent to the decay rate of the on-shell $b$ quark, which can be calculated in perturbation theory. Since experimental collaborations need to impose cuts on the kinematic variables, it is of great interest to consider differential decay distributions, from which it is possible to derive predictions for partial decay rates with arbitrary cuts.

At the NLO, the triple differential distribution of the decay $\overline{\mathrm{B}} \rightarrow X_{u} l \bar{v}_{l}$, together with several double and single differential distributions for the same process, have been calculated in [1].

Different frameworks were developed in order to account for the effect due to the experimental cuts In particular, in the shape function region, Soft Collinear Effective Thoery (SCET) provides an approriate approach for the evaluation of the triple-differential distribution of the inclusive semileptonic decay $\overline{\mathrm{B}} \rightarrow X_{u} l \bar{v}_{l}$. The NLO analysis of the latter process was presented [2]. At NNLO, the situation is more complicated, but the jet and soft function are known to $\mathscr{O}\left(\alpha_{S}^{2}\right)$ in perturbation theory. The hard function can be obtain from the two-loop QCD corrections to the decay of a $b$ quark into a $u$-quark and an off-shell $W$-boson [3]. The NNLO corrections to $\overline{\mathrm{B}} \rightarrow X_{u} l \bar{v}_{l}$, together with the determination of $\left|V_{u b}\right|$ from inclusive decays, have been done in [4]. From their analysis, the authors conclude that the NNLO corrections can be significant.

In this work, we focus on the analytic calculation of the NNLO corrections to the real emission of the decay process $b \rightarrow u W^{*}$, in the spirit of [1]. We follow the approach proposed in [5]. We use the Cutkosky rules to calculate the cut diagrams; the delta functions are replaced by the difference of two propagators with different causal $i 0^{+}$prescription. In so doing, the phase-space integrals can be evaluted in the same algorithmic way as in the multi-loop integral case. In particular, we use the Laporta algorithm [6], implemented in the computer program Reduze [7], for the reduction to the MIs, and the differential equations method [8] for their computation.

\section{NNLO QCD corrections: the real emission}

The contribution coming from the real emission at NNLO can be grouped in two different categories: two- and three-particle cut diagrams. So far we considered the three-particle cut diagrams.

After the reduction process, we find 12 MIs for the three-cut topologies, shown in Fig. 1. Five topologies have a single MI, two have two MIs, and one topology has three MIs.

Now we use the differential equation to solve the MIs. First we normalize the three-body space integral to 1 . The MIs satisfy a system of first-order linear differential equations in the dimenssionless variables $t=\sqrt{1-\frac{4 p^{2}}{m_{b}^{2} z^{2}}}$ and $z=-\frac{2 v \cdot p}{m_{b}^{2}}$, where $p$ and $v$ are the momentum of the $u$ and $b$ quark, respectively. A simple example is following:

$$
\frac{\partial}{\partial t} \sqrt{t}=\left[\frac{1}{t}+\frac{1}{1-t}-\frac{1}{t+1}+(D-4)\left(\frac{3}{2} \frac{1}{1-t}-\frac{3}{2} \frac{1}{t+1}+\frac{1}{t}\right)\right][
$$




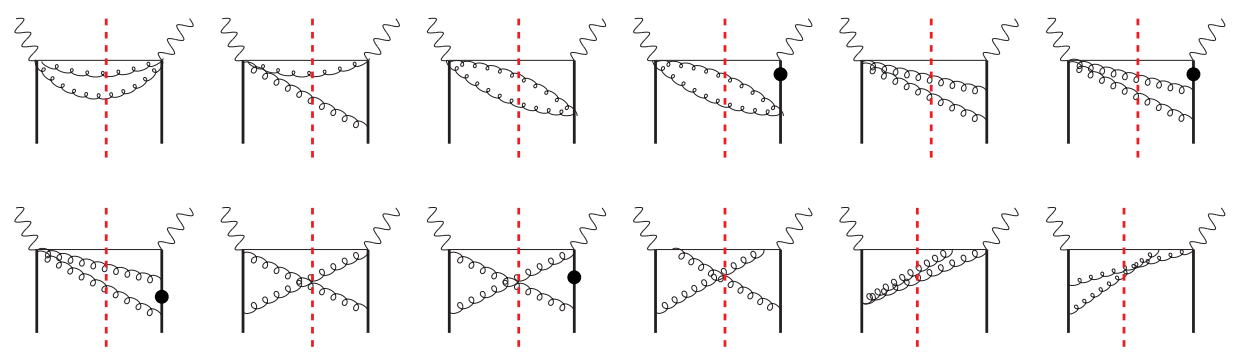

Figure 1: MIs for the three-particle cut diagrams. A dot on the propagator line means that the propagator is raised to the power 2. Bold lines represent a massive propagator. Thin and curly lines denote massless propagator.

$$
+\frac{1}{z m_{b}^{2}}\left[\frac{2}{1-t}-\frac{3}{2(1-t)}+\frac{2}{1+t}-\frac{4}{t}+(D-4)\left(\frac{3}{2(1+t)}-\frac{3}{t}\right)\right]
$$

The initial condition is found imposing the regularity of the function in $t=0$. We look for the solution as a Laurent series in $(D-4)$. In this case we find:

$$
\left[\begin{array}{l}
m_{b}^{2} z \\
t+1
\end{array}+\frac{1}{1-t}\right]+\mathscr{O}((D-4)) \text {. }
$$

\section{Conclusions}

We used the Laporta algorithm to perform the reduction to the MIs for the two- and threeparticle cut diagrams of the NNLO QCD correction to the decay procee $b \rightarrow u W^{*}$. We presented here the MIs of the three-particle cut set. We found at most three MIs per topology. The two-cut diagrams are under investigation.

\section{References}

[1] F. De Fazio and M. Neubert, JHEP 9906 (1999) 017.

[2] S. W. Bosch, B. O. Lange, M. Neubert, G. Paz, Nucl. Phys. B699, 335-386 (2004). B. O. Lange, M. Neubert, G. Paz, Phys. Rev. D72, 073006 (2005).

[3] R. Bonciani and A. Ferroglia, JHEP 0811 (2008) 065. H. M. Asatrian, C. Greub and B. D. Pecjak, Phys. Rev. D 78 (2008) 114028 . M. Beneke, T. Huber and X. Q. Li, Nucl. Phys. B 811 (2009) 77. G. Bell, Nucl. Phys. B 812 (2009) 264.

[4] C. Greub, M. Neubert, B. D. Pecjak, Eur. Phys. J. C65, 501-515 (2010).

[5] C. Anastasiou and K. Melnikov, Nucl. Phys. B 646 (2002) 220.

[6] S. Laporta, Int. J. Mod. Phys. A 15 (2000) 5087.

[7] C. Studerus, Comput. Phys. Commun. 181, 1293-1300 (2010).

[8] A.V. Kotikov, Phys. Lett. B 254 (1991) 158. Phys. Lett. B 259 (1991) 314. Phys. Lett. B 267 (1991) 123. E. Remiddi, Nuovo Cim. A 110 (1997) 1435. 